\title{
Human umbilical cord blood plasma alleviates age-related olfactory dysfunction by attenuating peripheral TNF- $\alpha$ expression
}

\author{
Byung-Chul Lee ${ }^{1, \#}$, Insung Kang ${ }^{1, \#}$, Seung-Eun Lee ${ }^{1}$, Jin Young Lee ${ }^{1}$, Nari Shin ${ }^{1}$, Jae-Jun Kim ${ }^{1}$, Soon Won Choi ${ }^{1}$ E \\ Kyung-Sun Kang ${ }^{1,2, *}$ \\ ${ }^{1}$ Adult Stem Cell Research Center and Research Institute for Veterinary Science, College of Veterinary Medicine, Seoul National \\ University, Seoul 08826, ${ }^{2}$ Institute for Stem Cell Regenerative Medicine, Kangstem Biotech CO., Seoul National University, Seoul 08826, \\ Korea
}

Social requirements are needed for living in an aging society and individual longevity. Among them, improved health and medical cares, appropriate for an aging society are strongly demanded. Human cord blood-derived plasma (hUCP) has recently emerged for its unique anti-aging effects. In this study, we investigated brain rejuvenation, particularly olfactory function, that could be achieved by a systemic administration of young blood and its underlying mechanisms. Older than 24-month-old mice were used as an aged group and administered with intravenous injection of hUCP repetitively, eight times. Anti-aging effect of hUCP on olfactory function was evaluated by buried food finding test. To investigate the mode of action of hUCP, brain, serum and spleen of mice were collected for further ex vivo analyses. Systemic injection of hUCP improved aging-associated olfactory deficits, reducing time for finding food. In the brain, although an infiltration of activated microglia and its expression of cathepsin $S$ remarkably decreased, significant changes of proinflammatory factors were not detected. Conversely, peripheral immune balance distinctly switched from predominance of Type 1 helper T (Th1) cells to alternative regulatory $T$ cells (Tregs). These findings indicate that systemic administration of hUCP attenuates age-related neuroinflammation and subsequent olfactory dysfunction by modulating peripheral immune balance toward Treg cells, suggesting another therapeutic function and mechanism of hUCP administration. [BMB Reports 2019; 52(4): 259-264]

*Corresponding author. Tel: +82-2-880-1246; Fax: +82-2-8767610; E-mail: kangpub@snu.ac.kr

${ }^{\text {"}}$ These authors contributed equally to this work.

https://doi.org/10.5483/BMBRep.2019.52.4.124

Received 4 June 2018, Revised 10 July 2018, Accepted 27 July 2018

Keywords: Cathepsin S, Immune balance, Microglia, Rejuvenation, Young blood

\section{INTRODUCTION}

Sense of smell is an essential sensory element for our daily life associated with survival and an important sense mediated with sensors related to flavor and memories. Functional impairment of the olfaction has significant impact on our safety, and physical and mental well-being. With other sensory systems, sense of smell declines with age affecting more than half of individuals between age 65 and 80 (1). Interestingly, severe olfactory deficits are often observed in early symptoms for several neurological disorders, including Alzheimer's disease (AD) (2) and Parkinson's disease (PD) (3), suggesting olfactory dysfunction as an early sign of neurodegeneration (4). Despite clear evidence on age- and neurodegeneration-related reduced olfactory function, pathological basis has not been explained and remains elusive.

Recently, there has been an emerging approach to understanding the relatively unappreciated communication that exists between the brain and systemic environment by uncovering regenerative activity in young blood with translational implications for aged liver, muscle, brain and other organs (5). In the brain, age-associated plasma chemokine GDF11 has been reported to increase neurogenesis in aged mice (6) while CCL11 is revealed to impair young brain function (7). Young blood including hUCP, reported to contain youth-associated proteins, such as TIMP2, GDF11, oxytocin, osteocalcin and Growth Hormone-Releasing Hormone $(\mathrm{GHRH})$, play unique roles for mediating anti-aging effects within specific organs. Young blood-mediated rejuvenation restores levels of remyelination of oligodendrocytes at the lesion site of aged spinal cord (8). It has been suggested that restoration mediated by peripheral expression of rejuvenation factors in a tissue-specific manner could improve brain functions and this possibility warrants further research (5). Given these therapeutic potentials, hUCP is currently in AMBAR (Alzheimer's Management by Albumin Replacement) and PLASMA (PLasma for Alzheimer's SymptoM Amelioration) clinical trials. 
Senescent cells are accumulated in tissues with age and display a senescence-associated secretory phenotype (SASP) that contribute in progress of aging through a non-cell autonomous mechanism (9). Increase of SASP-expressing senescent cells of non-neuronal origin from the brain is well reported in aging and age-related neurodegenerative diseases (10).

In this study, we investigated if systemic administration of hUCP could reverse age-related neuroinflammation and olfactory dysfunction and its underlying mechanisms.

\section{RESULTS}

\section{Aging leads to degenerative changes and secretion of proinflammatory cytokines as SASP}

The aging process accompanies several degenerative changes in normal physiological functions including hearing, smelling, growth of hair and immune response (11). Along with previous studies, we observed alopecia on back region of aged mice by gross examination (Fig. 1A). To confirm aged phenotype of old mice, we investigated expression level of aging biomarkers, MKP-1/DUSP1 (12) and p16 ${ }^{\text {INK4a }}$ (13). Expression level of MKP-1 and $116^{\text {INK4a }}$ increased in the spleen of aged mice (Fig. 1B). We additionally observed robust fat deposition, one of the aging-related physiological changes, in a peritoneal cavity of aged mice. Senescent cells secrete proinflammatory cytokines and chemokines developing SASP (14). We next measured serum level of IFN- $\gamma$, IL-6, MCP-1 and TNF secretion. Level of proinflammatory cytokines was significantly increased in the serum of aged mice (Fig. 1C). These results suggest that old
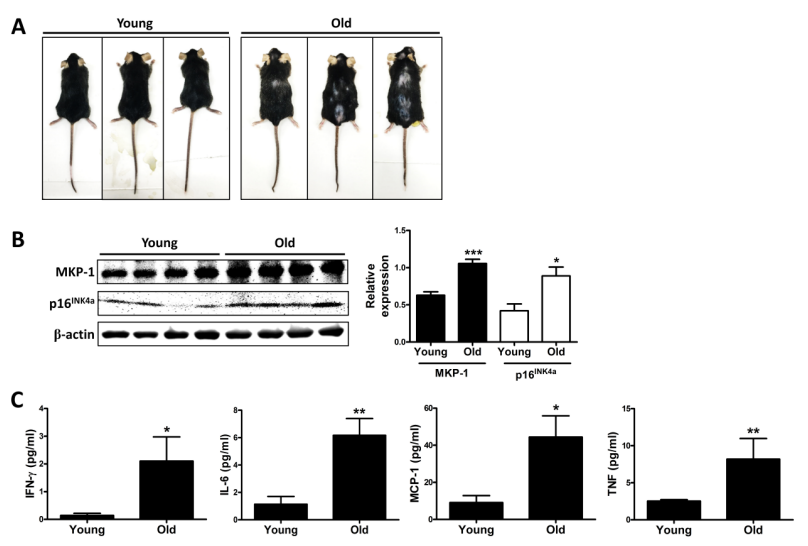

Fig. 1. Old mice represent aging-related phenotypes. 12-week-old mice and older than 24-month-old mice were used for in vivo experiments. (A) Gross images of young and aged mice. (B) Protein expression level of MKP-1/DUSP1 and $\mathrm{p} 16^{\mathrm{INK} 4 \mathrm{a}}$ in the spleen of mice was determined by Western blot analysis. (C) Secretion level of IFN- $\gamma$, IL-6, MCP- 1 and TNF in the serum of mice was determined by CBA analysis. $n=4-6$ mice per group. $* \mathrm{P}<0.05, * * \mathrm{P}<0.01, * * * \mathrm{P}<0.001$. Results are shown as the mean \pm SEM mice express aging-related physiological changes and increased secretion level of proinflammatory cytokines as SASP.

\section{hUCP alleviates aging-mediated olfactory dysfunction by suppressing the activation of microglia}

Aging impairs neurogenesis and stimulates microgliosis causing disorders in cognitive functions including learning and memory $(7,15)$. In addition, olfactory function declines in the process of aging (16). It is reported that systemic administration of hUCP improved aging-related impairments in cognitive function $(7,15)$. To assess if hUCP restores olfactory dysfunction in aged mice, we conducted the buried food finding test. Young mice found the buried food within three minutes and more than $50 \%$ of the mice started to eat the buried food within one minute. Conversely, five (of seven) aged mice failed to find the buried food within three minutes. However, latency was remarkably resolved by a systemic injection of hUCP (Fig. 2A and Supple. Vid. 1 to 3; 1:Young, 2:Old, 3: Old-hUCP). Activation of microglia is detrimental for the olfactory bulb $(\mathrm{OB})$ to function normally (17). We next examined activation of microglia by evaluating Iba1 expression in OB of young, aged and hUCP-injected aged

A
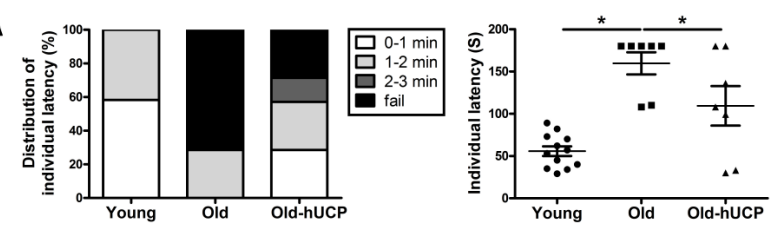

B
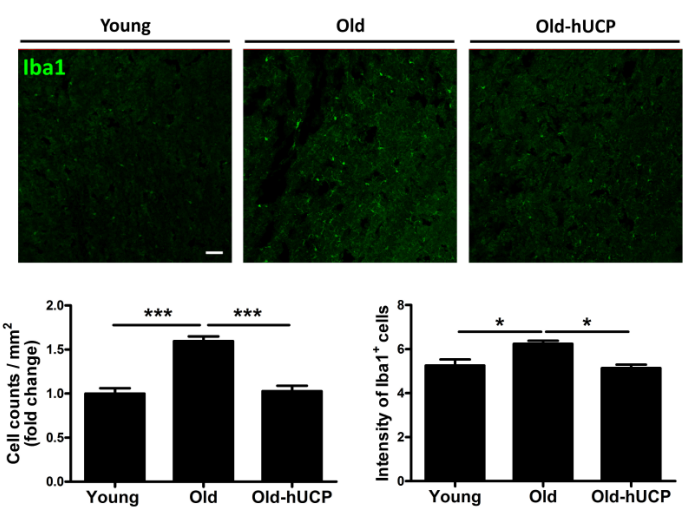

Fig. 2. hUCP improves the olfactory function by attenuating the activation of microglia. Aged mice were intravenously injected with $175 \mu \mathrm{l}$ of hUCP every three days for total eight times and neurological functions were addressed. (A) A stacked bar graph for the time-consuming pattern (left) and scattered dot graph of total elapsed time to find the buried food (right). (B) The level of microglial lba1 expression in the $\mathrm{OB}$ of mice were evaluated using immunohistochemistry. Bar $=50 \mu \mathrm{m}$. (C) Quantification of $\mathrm{Iba} 1^{+}$ cells and their fluorescent intensity. $\mathrm{n}=7-12$ mice per group. ${ }^{*} \mathrm{P}$ $<0.05, * * * P<0.001$. Results are shown as the mean \pm SEM. 
mice. In the $\mathrm{OB}$ of aged mice, the number of $\mathrm{Iba} 1^{+}$cells and intensity of cells significantly increased. Infiltration of lba $1^{+}$ cells and intensity of cells were retrieved by intravenous injection of hUCP (Fig. 2B and C). Together, aging-mediated impairment of olfactory function is alleviated by hUCP infusion that inhibits microglial activation.

\section{Systemic administration of hUCP inhibits TNF- $\alpha$-mediated} microglial activation by regulating expression of cathepsin $S$ Cathepsin S contributes to microglia-mediated olfactory dysfunction through activation of the immune system in the brain (18). To determine if activation of microglia in the brain of aged mice could be attenuated by systemically infused hUCP, we determined expression level of cathepsin $S$ in the $\mathrm{OB}$ of mice by Western blot analysis. Consistent with previous results, expression level of cathepsin S significantly increased in aged OB. Importantly, activation of cathepsin S was restored by hUCP injection (Fig. 3A). Factors that influence brain aging remain unclear. To address these issues, we next treated BV-2 microglial cells (Fig. S1) with IFN- $\gamma$, IL-6, MCP-1 and TNF- $\alpha$ to assess which cytokine caused neuroinflammation. All cytokines did not reveal significant change but only TNF- $\alpha$ increased level of cathepsin $\mathrm{S}$ expression of BV-2 cells (Fig. 3B). In addition, activation of BV2 cells verified by increased secretions of NO, MCP-1, IL-6 and TNF (Fig. S2). However, we did not detect immunologic changes including the level of TNF- $\alpha$ in aged OB by hUCP infusion (data not shown). Modulation of peripheral immune response can protect the brain from various neurologic risks $(19,20)$. To determine if administration of hUCP attenuates systemic proinflammatory immune milieu, we measured the secretion

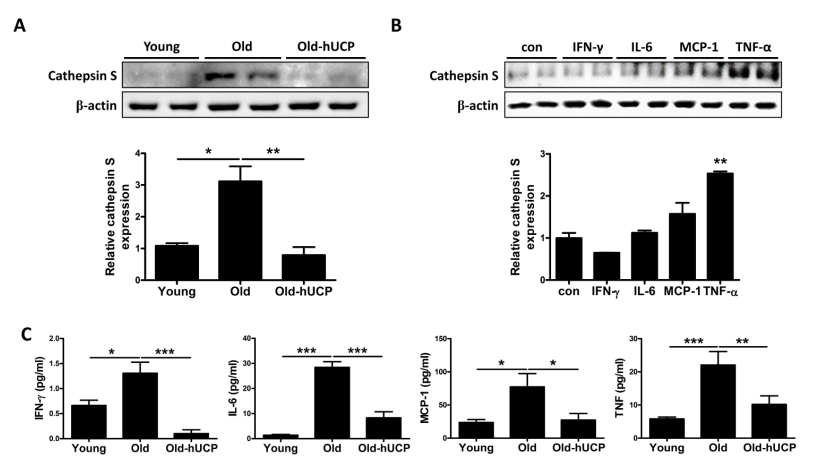

Fig. 3. hUCP recovers the microglial expression of cathepsin $S$ in $\mathrm{OB}$ and TNF- $\alpha$ induces microglial activation. Aged mice were intravenously injected with $175 \mu \mathrm{l}$ of hUCP every three days for total eight times and microglial activation was addressed. (A) The level of cathepsin $\mathrm{S}$ in the OB was measured by Western blot analysis. (B) After indicated treatments, expression level of cathepsin $\mathrm{S}$ on BV-2 cells was measured by Western blot analysis. (C) The level of IFN- $\gamma$, IL-6, MCP-1 and TNF in serum of mice was measured by $\mathrm{CBA}$ analysis. $\mathrm{n}=4$ mice per group. $* \mathrm{P}<0.05, * * \mathrm{P}<0.01, * * * \mathrm{P}<0.001$. Results are shown as the mean \pm SEM level of proinflammatory cytokines in the serum of mice. Aging-related increased secretion of IFN- $\gamma$, IL-6, MCP-1 and TNF remarkably recovered in the serum after systemic administration of hUCP (Fig. 3C). Together, hUCP has a role in inhibiting activation of microglia in $\mathrm{OB}$ by suppressing indirectly age-related increase of TNF- $\alpha$.

\section{Administration of hUCP resolves systemic inflammation in aged mice via promotion of Treg cells and IL-10 secretion} We further investigated if hUCP infusion could alter immune cell composition in spleen. Spleen of aged mice significantly enlarged and age-induced splenomegaly recovered by systemic infusion of hUCP (Fig. 4A). Gene expression level of IFN- $\gamma$ and TNF- $\alpha$, crucial factors for proinflammatory function of $\mathrm{T}$ helper type 1 (Th1) cells, increased in spleen of aged mice. Increased level of IFN- $\gamma$ and TNF- $\alpha$ was dramatically retrieved by hUCP infusion (Fig. 4B). Similar to previous results, transcription factor T-Box (TBX) 21 was highly expressed in aged mice and expression decreased by injection of hUCP (Fig. 4B). IL-10 prevents age-related inflammation and production is dysregulated with aging. In the same context, expression level of IL-10 declined in the spleen of aged mice. In addition, hUCP-infused mice presented significantly increased gene expression level of IL-10 (Fig. 4B). We further assessed a proportion of most prominent IL-10 producing cells, $\mathrm{CD} 4{ }^{+} \mathrm{CD} 25^{+} \mathrm{FoxP}^{+}$Treg cells in the spleen. As expected, the proportion of $\mathrm{CD}^{+}{ }^{+} \mathrm{CD} 25^{+} \mathrm{FoxP}^{+}$Treg cells markedly increased in the spleen of hUCP-injected mice compared to young and aged mice (Fig. 4C). Findings indicate that systemically infused hUCP exerts anti-inflammatory effects by regulating immune balance between Th1 and Treg cells.

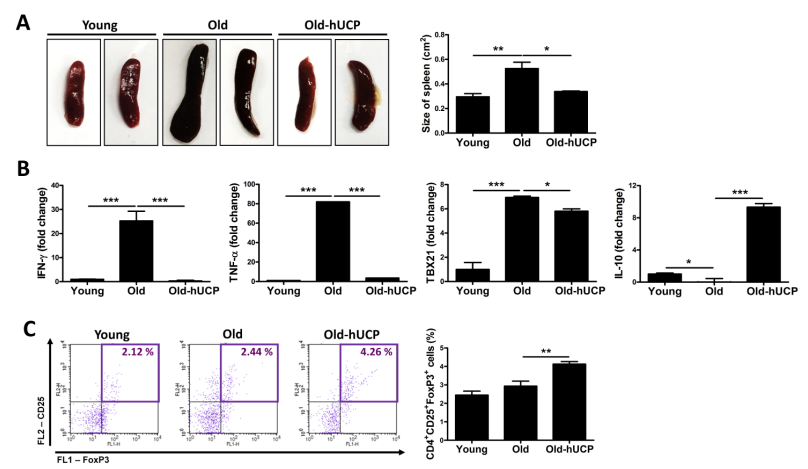

Fig. 4. hUCP suppresses age-related increase of peripheral inflammation through modulation of immune balance between Th1 and Treg cells. Aged mice were intravenously injected with $175 \mu \mathrm{l}$ of hUCP every three days for total eight times and systemic immune functions were addressed. (A) Representative gross images and size of the spleen. (B) Gene expression level of IFN- $\gamma$, TNF- $\alpha$, TBX21 and IL-10 was determined by qRT-PCR. (C) Population of $\mathrm{CD} 4{ }^{+} \mathrm{CD} 25^{+}$FoxP3 $^{+}$cells in spleen was analyzed by flow cytometric analysis. $\mathrm{n}=5-6$ mice per group. ${ }^{*} \mathrm{P}<0.05,{ }^{*} * \mathrm{P}<$ $0.01, * * * \mathrm{P}<0.001$. Results are shown as the mean \pm SEM. 


\section{DISCUSSION}

Recently, diverse strategies, including an application of cord blood-derived plasma, have been proposed to attenuate aging-associated functional disorders. Most of the studies focused on elucidating an anti-aging effect of developed agents or investigating the therapeutic mechanism only in a neurological aspect. In this study, we have demonstrated that the injection of plasma isolated from umbilical cord blood suppressed neuroinflammation and restored olfactory dysfunction by modulating systemic immune milieu.

In the aging process, senescent cells in the body display distinctive secretory phenotype, namely SASP, that secretes robust amount of proinflammatory cytokines (21). Consistent with previous studies, we determined the level of representative proinflammatory cytokines, IFN- $\gamma$, IL-6, MCP-1 and TNF significantly increased in the serum of aged mice. In addition, we also confirmed aged phenotype by detecting upregulated expression level of aging biomarker, MKP-1/DUSP1 in spleen of old mice. Although MKP-1/DUSP1 protects brain tissues against neuroinflammation (22) and regulate pro- and anti-inflammatory responses (23), it conversely plays a critical role in proliferation and activation of T cells (24). It suggests that age-related upregulation of MKP-1/DUSP1 in spleen may be related to increased production of proinflammatory cytokines.

Aging of the brain exhibits diverse neurodegenerative changes related to cognitive deficits, learning disability and dementia (25). Among these uncomfortable changes, olfactory dysfunction is an important indicator because every process involved with proliferation, maturation, integration and apoptosis of olfactory neurons should be fine-tuned to provide olfactory function (26). We previously explained microgliosis contributed to progression of olfactory deficits in Niemann pick type C1 mice by increasing neuronal apoptosis (17). In this study, we extend our finding of microglial dysregulation on the aged mice to explain age-related decline of olfaction. To reverse aging-associated olfactory dysfunction, we used the systemic injection of hUCP and achieved similar results. It has been demonstrated that hippocampal function could be revitalized by administration of hUCP by Wyss-coray group (15). Additionally, hUCP stimulates neurogenesis in the aged brain (27). Similar to previous studies, hUCP could ameliorate age-related olfactory deficits.

Immune system undergo a shift to a mild, but chronic inflammatory state, wherein secretion of TNF- $\alpha$ and IL- $1 \beta$ is increased in the CNS, by aging (28). As microglia participate as a specialized immune cell of the CNS, these age-associated alterations in proinflammatory cytokines may impair the normal functional role of microglia. Upregulated activation markers including MHC II, CD86 and TLRs, nucleotidebinding oligomerization domain-like (NOD)-like receptors are examined in aged microglia (29). As the brain ages, microglia become more inflammatory and resistant to anti-inflammatory signals (30). Consistent with these studies, we found that the number of Iba1-activated microglia significantly increased in the aged brain and microglial activation was restored by systemically injected hUCP.

To explore specific mechanism underlying the antiinflammatory effect of hUCP, we investigated expression level of cathepsin S in the brain and microglia cell. Cathepsin S, a member of the cysteine cathepsins, has an important function associated with the MHC class II molecule affecting bone marrow derived antigen presenting cells such as dendritic cells, macrophages and B cells (31). In the aged brain, the level of cathepsin $\mathrm{S}$ is also increased in a number of cathepsin S-expressing cells including microglia (32). Increased cathepsin S level in the aged brain could strongly effect olfactory deficits and other microglia-dependent neuropathies. In recent studies associated with brain pathogenesis, evidence suggests that cathepsin $\mathrm{S}$ plays a major role in inflammatory processes. In our previous study, we suggested cathepsin S as a key regulator in development of microglial neurotoxicity (18). Activated microglia release cathepsins to induce neuronal death through degradation of extracellular matrix proteins (33). As expected, we determined that upregulated expression of cathepsin $\mathrm{S}$ in the aged brain was resolved by hUCP. More interestingly, BV-2 microglial cells only responded to TNF- $\alpha$, but not the other treatments, resulting in expression of cathepsin S. Although many studies have suggested that excessive TNF- $\alpha$ expression was involved in neurodegenerative disorders, we couldn't identify significant immunologic changes including TNF- $\alpha$ expression in the brain.

Of note, peripheral immune system and CNS are closely connected and reciprocally cooperated to maintain a homeostasis (34). In addition, neurological functions such as stress, pain and cognition could be influenced by peripheral immune response (35). Among proinflammatory cytokines, peripheral administration of TNF- $\alpha$ can induce neuroinflammation and functional disorders (36). Systemicallyinjected anti-TNF reagent ameliorates cerebral neurodegeneration (19). In this study, we also demonstrated that systemic administration of hUCP suppressed TNF- $\alpha$ secretion. Progressive deterioration of immune function is the well-defined phenotype of human aging (37). Although IL-10 plays a crucial role in resolution of aging-associated inflammation (38), production and secretion of IL-10 are dysregulated with aging (39). Additionally, it has been clear that Treg cells with functional disorder accumulate in the organs of aged individuals and the balance between immune effector cells such as Th17 cells and regulatory cells is disturbed (40). According to our results, the IL-10 expression and infiltration of Treg cells markedly increased after hUCP transfusion. It illustrates that systemic administration of hUCP recovers the function of Treg cells and stimulated cells to secrete an anti-inflammatory cytokine, IL-10 to inhibit Th1 response and consequent TNF- $\alpha$ secretion. With this potential, 
hUCP may be administered to other diseases, that present dysregulated cytokine network and immune balance.

Together, this study revealed that systemic application of hUCP suppressed microglial activation by regulating peripheral immune balance between Th1 and Treg cells, consequently alleviated olfactory dysfunction mediated by senescence-related neuroinflammation.

\section{MATERIALS AND METHODS}

\section{Animals}

Animal experiments were conducted in accordance with approved guidelines of Seoul National University Institutional Animal Care and Use Committee (IACUC No. SNU-180430-6). Male C57BL/6 mice (Orientbio, Sungnam, Republic of Korea) were housed in the animal facility of Seoul National University. Young (12-week-old) and aged (older than 24-month-old) mice were used for experiments. After intravenous application of hUCP, neurological function of mice was assessed. The mice did not represent immune rejection-related signs, including sudden death, weight loss and inflammation, caused by a xenotransplantation. After the mice were anesthetized, brain, spleen and blood samples were collected for more ex vivo examinations.

\section{Isolation and injection of hUCP}

All experiments involved in hUCP were approved by Institutional Review Board (IRB) of the Boramae Hospital and Seoul National University (IRB No. 1707/003-006) with informed maternal consent. Injection and isolation of hUCP followed Castellano and colleague's method (15). Briefly, cord blood was collected immediately after birth and centrifuged at $1,000 \mathrm{~g}$ for 10 minutes at $4^{\circ} \mathrm{C}$ for isolation of plasma. Isolated plasma was stored aliquots at $-80^{\circ} \mathrm{C}$ until treatments. $175 \mu \mathrm{l}$ of hUCP was administered intravenously every three days for total eight times.

\section{Buried food finding test}

The buried food test was used to evaluate olfaction on young and old mice. Mice from young and old experimental group were fasted for 24 hours before the test. Before the nasal test, the mice were individually habituated for 10 minutes in a new cage with fresh bedding. While the mice were going through habituation step, a piece of standard chow $(1 \times 1 \mathrm{~cm})$ was buried under the bedding in the middle of a new test cage with a depth of $0.5 \mathrm{~cm}$. After the habituation step, the subject was placed in the left corner of the test cage and allowed to move freely to find the hidden food for three minutes. During the test, all activities including digging and sniffing were recorded until the mouse discovered the standard chow. After the test, each of the mice were placed on the bedding with food placed on the top (exposed food test) to ensure the buried food test was based on olfaction and not on visual ability.

\section{Statistical analysis}

Mean values of all results were expressed as the mean \pm SEM. Statistical analyses were conducted using Student's 2-tailed t-test or one-way ANOVA followed by Bonferroni post-hoc test for multigroup comparisons using GraphPad Prism version 5.0 (GraphPad Software, San Diego, CA, USA). Statistical significance is indicated in the figure legend.

\section{ACKNOWLEDGEMENTS}

This study was supported by the National Research Foundation of Korea (NRF) grant funded by the Korea government (MSIT) (No. 2018R1A2B3008483) and a grant of the Korea Health Technology R\&D Project through the Korea Health Industry Development Institute (KHIDI), funded by the Ministry of Health \& Welfare, Republic of Korea (grant number: HI18C0421).

\section{CONFLICTS OF INTEREST}

The authors have no conflicting interests.

\section{REFERENCES}

1. Duffy VB, Backstrand JR and Ferris AM (1995) Olfactory dysfunction and related nutritional risk in free-living, elderly women. J Acad Nutr Diet 95, 879-884

2. Ruan Y, Zheng XY, Zhang HL, Zhu W and Zhu J (2012) Olfactory dysfunctions in neurodegenerative disorders. J Neurosci Res 90, 1693-1700

3. Doty RL, Stern MB, Pfeiffer C, Gollomp SM and Hurtig HI (1992) Bilateral olfactory dysfunction in early stage treated and untreated idiopathic Parkinson's disease. J Neurol Neurosurg Psychiatry 55, 138-142

4. Albers MW, Tabert MH and Devanand D (2006) Olfactory dysfunction as a predictor of neurodegenerative disease. Curr Neurol Neurosci Rep 6, 379-386

5. Castellano JM, Kirby ED and Wyss-Coray T (2015) Blood-borne revitalization of the aged brain. JAMA Neurol 72, 1191-1194

6. Katsimpardi L, Litterman NK, Schein PA et al (2014) Vascular and neurogenic rejuvenation of the aging mouse brain by young systemic factors. Science 344, 630-634

7. Villeda SA, Luo J, Mosher KI et al (2011) The ageing systemic milieu negatively regulates neurogenesis and cognitive function. Nature 477, 90-94

8. Ruckh JM, Zhao J-W, Shadrach JL et al (2012) Rejuvenation of regeneration in the aging central nervous system. Cell Stem Cell 10, 96-103

9. Robbins PD (2017) Extracellular vesicles and aging. Stem Cell Investig 4, 98

10. Chinta SJ, Woods G, Rane A, Demaria M, Campisi J and Andersen JK (2015) Cellular senescence and the aging brain. Exp Gerontol 68, 3-7

11. Lopez-Otin C, Blasco MA, Partridge L, Serrano M and Kroemer G (2013) The hallmarks of aging. Cell 153, 1194-1217 
12. Flach RJ and Bennett AM (2010) MAP kinase phosphatase-1-a new player at the nexus between sarcopenia and metabolic disease. Aging (Albany NY) 2, 170-176

13. Collado M, Blasco MA and Serrano M (2007) Cellular senescence in cancer and aging. Cell 130, 223-233

14. Young AR and Narita M (2009) SASP reflects senescence. EMBO Rep 10, 228-230

15. Castellano JM, Mosher KI, Abbey RJ et al (2017) Human umbilical cord plasma proteins revitalize hippocampal function in aged mice. Nature 544, 488

16. Kovács T (2004) Mechanisms of olfactory dysfunction in aging and neurodegenerative disorders. Ageing Res Rev 3, 215-232

17. Seo Y, Kim H-S, Shin Y et al (2014) Excessive microglial activation aggravates olfactory dysfunction by impeding the survival of newborn neurons in the olfactory bulb of Niemann-Pick disease type C1 mice. Biochim Biophys Acta Mol Basis Dis 1842, 2193-2203

18. Seo Y, Kim HS, Kang I et al (2016) Cathepsin S contributes to microglia-mediated olfactory dysfunction through the regulation of $\mathrm{Cx} 3 \mathrm{Cl} 1-\mathrm{Cx} 3 \mathrm{cr} 1$ axis in a Niemann-Pick disease type C1 model. Glia 64, 2291-2305

19. Clausen BH, Degn M, Martin NA et al (2014) Systemically administered anti-TNF therapy ameliorates functional outcomes after focal cerebral ischemia. J Neuroinflammation 11, 203

20. Hennessy E, Gormley S, Lopez-Rodriguez AB, Murray C, Murray C and Cunningham C (2017) Systemic TNF-alpha produces acute cognitive dysfunction and exaggerated sickness behavior when superimposed upon progressive neurodegeneration. Brain Behav Immun 59, 233-244

21. Freund A, Patil CK and Campisi J (2011) p38MAPK is a novel DNA damage response-independent regulator of the senescence-associated secretory phenotype. EMBO J 30, 1536-1548

22. Eljaschewitsch E, Witting A, Mawrin C et al (2006) The endocannabinoid anandamide protects neurons during CNS inflammation by induction of MKP-1 in microglial cells. Neuron 49, 67-79

23. Chi H, Barry SP, Roth RJ et al (2006) Dynamic regulation of pro-and anti-inflammatory cytokines by MAPK phosphatase 1 (MKP-1) in innate immune responses. Proc Natl Acad Sci U S A 103, 2274-2279

24. Zhang Y, Reynolds JM, Chang SH et al (2009) MKP-1 is necessary for $\mathrm{T}$ cell activation and function. J Biol Chem 284, 30815-30824

25. Hajek A, Brettschneider C, Luhmann D et al (2016) Effect of Visual Impairment on Physical and Cognitive Function in Old Age: Findings of a Population-Based Prospective
Cohort Study in Germany. J Am Geriatr Soc 64, 2311-2316

26. Nissant A and Pallotto M (2011) Integration and maturation of newborn neurons in the adult olfactory bulb-from synapses to function. Eur J Neurosci 33, 1069-1077

27. Bachstetter AD, Pabon MM, Cole MJ et al (2008) Peripheral injection of human umbilical cord blood stimulates neurogenesis in the aged rat brain. BMC Neurosci 9, 22

28. Streit WJ, Sammons NW, Kuhns AJ and Sparks DL (2004) Dystrophic microglia in the aging human brain. Glia 45 , 208-212

29. Letiembre $M$, Hao $W$, Liu $Y$ et al (2007) Innate immune receptor expression in normal brain aging. Neurosci 146, 248-254

30. Nissen JC (2017) Microglial function across the spectrum of age and gender. Int J Mol Sci 18, 561

31. Kirschke H (2013) Cathepsin S; in Handbook of Proteolytic Enzymes (Third Edition). Elsevier 1824-1830

32. Wendt W, Lübbert H and Stichel CC (2008) Upregulation of cathepsin $\mathrm{S}$ in the aging and pathological nervous system of mice. Brain Res 1232, 7-20

33. Nakanishi $H$ (2003) Microglial functions and proteases. Mol Neurol 27, 163-176

34. Veiga-Fernandes $\mathrm{H}$ and Artis D (2018) Neuronal-immune system cross-talk in homeostasis. Science 359, 1465-1466

35. Maier SF (2003) Bi-directional immune-brain communication: Implications for understanding stress, pain, and cognition. Brain Behav Immun 17, 69-85

36. Biesmans S, Bouwknecht JA, Ver Donck L et al (2015) Peripheral Administration of Tumor Necrosis Factor-Alpha Induces Neuroinflammation and Sickness but Not Depressive-Like Behavior in Mice. Biomed Res Int 2015, 716920

37. Raynor J, Lages CS, Shehata H, Hildeman DA and Chougnet CA (2012) Homeostasis and function of regulatory $\mathrm{T}$ cells in aging. Curr Opin Immunol 24, 482-487

38. Dagdeviren S, Jung DY, Friedline RH et al (2017) IL-10 prevents aging-associated inflammation and insulin resistance in skeletal muscle. FASEB J 31, 701-710

39. Spencer NF, Norton SD, Harrison LL, Li GZ and Daynes RA (1996) Dysregulation of IL-10 production with aging: possible linkage to the age-associated decline in DHEA and its sulfated derivative. Exp Gerontol 31, 393-408

40. Schmitt V, Rink L and Uciechowski P (2013) The Th17/Treg balance is disturbed during aging. Exp Gerontol 48, 1379-1386 